\title{
Sensitivity Improvement of Photo-Ionization Detectors by Coating Carbon Nanotubes on Electrodes
}

\author{
Soonjae Pyo, Kyounghoon Lee, Taegyoon Noh, Jongbaeg Kim* \\ School of Mechanical Engineering, Yonsei University, \\ 50 Yonsei-ro, Seodaemun-gu, Seoul 03722, Republic of Korea \\ kimjb@yonsei.ac.kr
}

\begin{abstract}
:
This paper reports the effect of carbon nanotubes (CNTs) on metal electrodes of photo-ionization detectors (PIDs) for volatile organic compounds (VOCs) for the first time. The CNTs on the electrodes widen an adsorption surface area and improve an adsorption property of electrodes for PIDs, which leading to more effective interaction with ionized VOCs and electrons. The CNTs also increase the local electric field density, enhancing the ion-capture performance. Compared to the conventional metal electrodes, the CNTs-coated electrodes resulted in over $50 \%$ enhancement of responsiveness for an identical concentration of toluene (1000 ppm), showing the possibility of highly sensitive PIDtype VOC sensors.
\end{abstract}

Key words: Photo-ionization detector, Carbon nanotube, Volatile organic compounds, UV light, Room temperature gas sensor

\section{Introduction}

VOCs are well known for causing photochemical smog as well as respiratory distress, skin diseases, and cancer to humans [1]. While numerous studies on detecting the concentration of VOCs have been researched, photo-ionization is one of the few promising VOC detection methods. It is advantageous in selectivity, response time, and insensitivity to humidity or temperature change [2]. Fig.1 shows the detection mechanism of PIDs. VOCs ionized by short wavelength UV light are separated into electrons and positively charged ions. The electrons and ions are then captured by polarized electrodes placed nearby the ionizer, generating current flow through the electrodes. At low concentration of VOC, however, this method typically results in small signal with large noise and thus many studies were reported to enhance the signal responsiveness. One example is to change the shape of the electrodes for PIDs into an interdigitated form for larger area of interaction between the electrodes and ions [3].

\section{Results and Discussion}

The fundamental idea in this work is to further enhance the adsorption surface area and adsorption property of electrodes for PIDs by introducing CNTs on top of the electrodes.

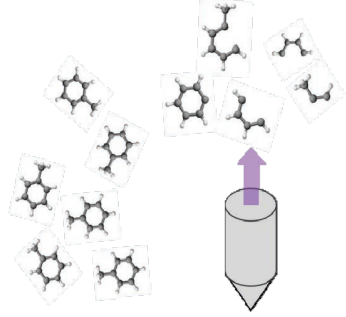

(a) Introducing toluene

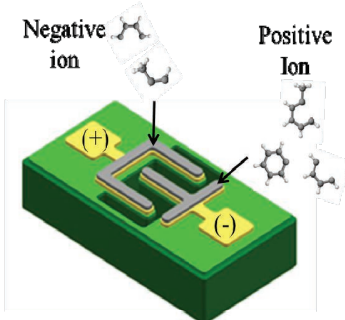

(c) Placing the ion to each electrode \& changing amount of current
Fig. 1. A schematic diagram to show PID-type toluene-sensing mechanism. (a) Introducing toluene gas into the chamber, (b) lonizing toluene gas into positively and negatively charged ions by UV source, (c) Capturing the ions using polarized electrodes.

CNTs have a high surface to volume ratio as well as high electrical conductivity, thus the electrodes coated with CNTs have a better chance to interact with ionized VOCs and electrons than the simple metal electrodes. In addition to the larger area, various functional groups created on the surface of CNTs during the synthesis and the purification process for CNTs would help the electrodes to capture the ionized VOCs and electrons. The CNTs on flat electrodes will increase the local electric field density, which could also enhance the ioncapture performance. The fabrication process given in Fig. 2 started on $\mathrm{SiO}_{2} / \mathrm{Si}$ substrate. A 

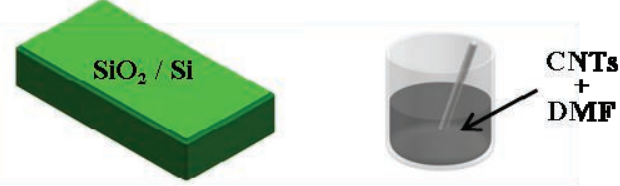

(a) Preparing a substrate
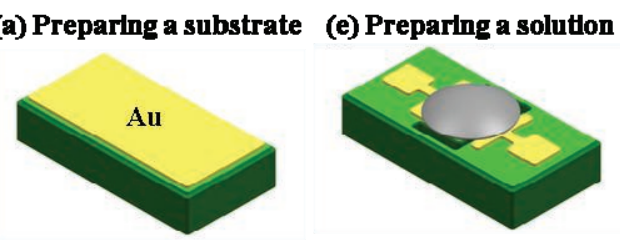

(b) Depositing

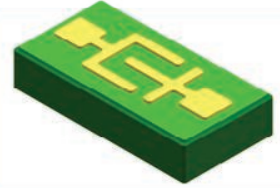

(c) Patterning

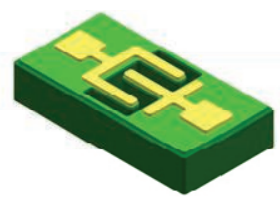

(d) Deep reacttve-lon etching

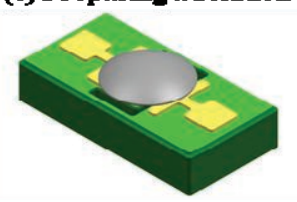

(f) Drop-casting

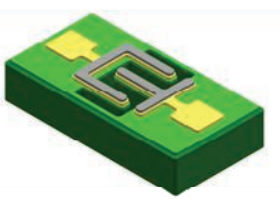

(g) Evaporating

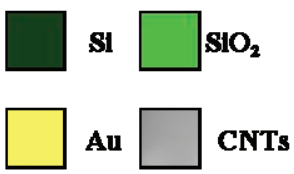

Fig. 2. The fabrication process for the electrodes with CNTs. (a)Preparing a $\mathrm{SiO}_{2} / \mathrm{Si}$ substrate, (b)Depositing a gold film, (c)Patterning the gold film, (d)Deep reactive-ion etching of silicon, (e)Preparing CNTs/DMF solution by sonication, (f) Drop-casting the solution on the electrodes, (g) Evaporating the solvent.
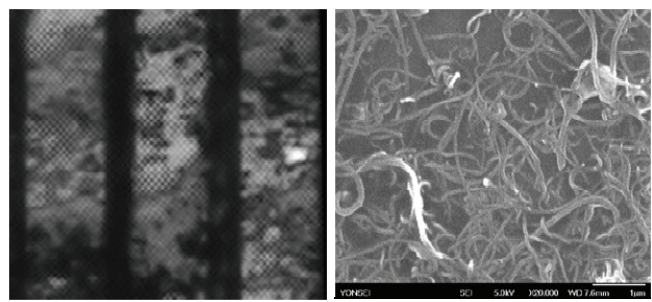

Fig. 3. (a) Optical image of gold electrodes with CNTs coated, (b) Magnified SEM image of CNTs coated on gold electrodes.

gold film is coated by metal evaporation and patterned. The channel through which the VOC gas and light would pass is fabricated on the $\mathrm{Si}$ substrate by deep reactive-ion etching. After this, the CNT solution that consists of $300 \mathrm{mg}$ multi-walled CNTs and $10 \mathrm{~g}$ dimethylformamide is drop-casted on the electrodes. Fig. 3 presents an optical image and a SEM image of the electrodes coated with CNTs. Fig. 4 depicts the experimental setup for PID of VOC, the toluene in this work. The polarization voltage from a DC supply and the current flow was measured by an ammeter. Time-responses of the measured current from PID with the CNTcoated electrodes (red line) and without CNTs (black line) are compared in Fig. 5. The presented current changes were normalized by

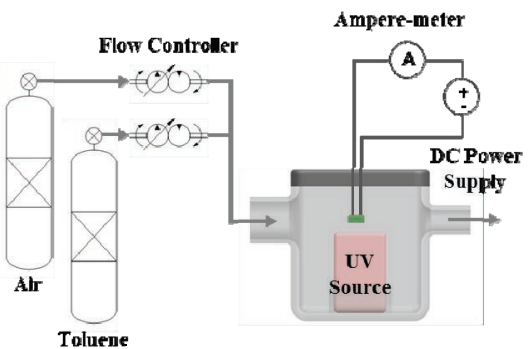

Fig. 4. Schematic diagram of the experimental setup.

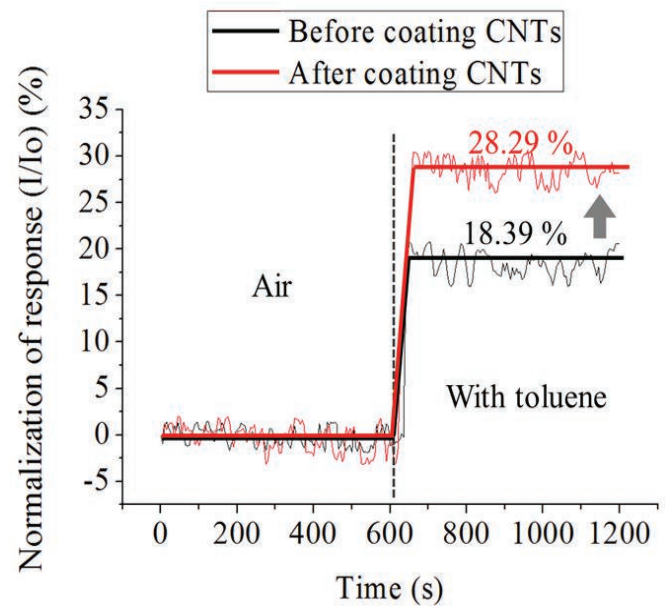

Fig. 5. Time-responses of the measured current from PID with the CNT-coated electrodes (red line) and without CNTs (black line).

dividing by the initial current, and the current change of $18.38 \%$ from simple metal electrodes increased to $28.29 \%$ for the CNTs-coated electrode under an identical concentration of toluene at $1000 \mathrm{ppm}$.

\section{Acknowledgements}

This material is based upon work supported by the Ministry of Trade, Industry \& Energy(MOTIE, Korea) under Industrial Technology Innovation Program. No.10054548, 'Development of Suspended Heterogeneous Nanostructure-based Hazardous Gas Microsensor System'

\section{References}

[1] B. Huang, C. Lei, C. Wei, G. Zeng, Chlorinated volatile organic compounds (Cl-VOCs) in environment sources, potential human health impacts and current remediation technologies, Environmental International 71, 118-138 (2014); doi: 10.1016/j.envint.2014.06.013

[2] M. Suresh, N. J. Vasa, V. Agarwal, J. Chandapillai, UV photo-ionization based asymmetric field differential ion mobility sensor for trace gas detection, Sensors and Actuators B: Chemical 195, 44-51 (2014); doi: 10.1016/j.snb.2014.01.008

[3] P. N. Luke, Single-polarity charge sensing in ionization detectors using coplanar electrodes, Applied Physics Letters 65, 2884 (1994); doi: 10.1063/1.112523 